\title{
Understanding the Connection Between Self-Esteem and Aggression: The Mediating Role of Emotion Dysregulation
}

\author{
Carlo Garofalo $^{1 *}$, Christopher J. Holden ${ }^{2}$, Virgil Zeigler-Hill ${ }^{2}$, and Patrizia Velotti ${ }^{3}$ \\ ${ }^{1}$ Department of Dynamic and Clinical Psychology, Sapienza University of Rome, Rome \\ ${ }^{2}$ Oakland University, Rochester, Michigan \\ ${ }^{3}$ Department of Educational Sciences, University of Genoa, Genoa
}

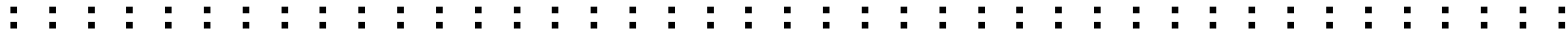

The purpose of the present study was to extend previous knowledge concerning the link between self-esteem and aggression by examining the mediating role of emotion dysregulation among offenders and community participants. A sample of 153 incarcerated violent offenders and a community sample of 197 individuals completed self-report measures of self-esteem level, emotion dysregulation, and trait aggression. Offenders reported lower levels of self-esteem than community participants, as well as greater levels of emotional nonacceptance and hostility. Bootstrapping analyses were performed to test whether emotion dysregulation mediated the association between self-esteem level and aggression. In the offender sample, mediation models were significant for three of the four aspects of trait aggression that were considered. Emotion dysregulation fully mediated the links that low self-esteem had with physical aggression, anger, and hostility. The same pattern (with the addition of full mediation for verbal aggression) was confirmed in the community sample. Our findings suggest that emotion dysregulation may play an important role in the connection between low self-esteem and aggression. Alternative models of the associations among these variables were tested and discussed. As a whole, the present results are consistent with those of other studies and suggest that it may be beneficial to include emotion regulation modules as part of prevention and treatment programs for violent offenders. Aggr. Behav. 9999:1-13, 2015. @ 2015 Wiley Periodicals, Inc.

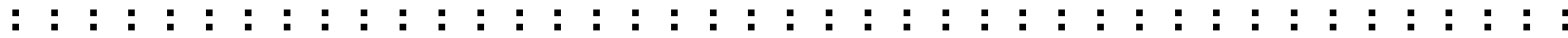

Keywords: self-esteem; emotion dysregulation; aggression; offenders

\section{INTRODUCTION}

Previous research concerning aggression has focused almost exclusively on aggressive behavior and attempted to categorize this using dichotomies such as impulsive versus premeditated aggression (e.g., Stanford et al., 2003). Although it certainly has benefits, this approach may have hindered a more complete understanding of the motives underlying human aggression (Bushman \& Anderson, 2001). Broadening the focus of aggression research to include the study of the psychological characteristics displayed by individuals who tend to behave aggressively has made it possible to further explore cognitive and emotional aspects of aggression (Hoeve et al., 2015). For example, Buss and Perry (1992) developed an influential framework that emphasized the importance of individual differences and psychological functioning in the conceptualization of aggression by focusing on three underlying components: cognitive (e.g., hostile thoughts), emotional (e.g., angry feelings), and behavioral (e.g., aggressive actions).

Research concerning the role that self-esteem has as an antecedent of aggression has led to considerable debate with several authors reporting evidence for a link between low self-esteem and aggression (Boden, Fergusson, \& Horwood, 2007; Donnellan, Trzesniewski, Robins, Moffitt, \& Caspi, 2005; Walker \& Bright, 2009) whereas others argue that it is high levels of self-esteem that may trigger aggressive behavior (Bushman et al., 2009; Muller, Bushman, Subra, \& Ceaux, 2012). Overall, a recent meta-analysis (Rosenthal, Montoya, Ridings, Rieck, \& Hooley, 2011) found an average negative correlation $(r=-.29,95 \%$ CI $[-.24,-.33])$ between self-esteem and aggression/anger, as measured using either the Aggression Questionnaire (Buss \& Perry,

${ }^{*}$ Correspondence to: Carlo Garofalo, Sapienza University of Rome, Department of Dynamic and Clinical Psychology, Via degli Apuli, 1, 00185, Rome, Italy.

E-mail: carlo.garofalo@uniroma1.it; cgarofalo24@gmail.com

Received 29 December 2014; Revised 24 May 2015; Accepted 1 June 2015

DOI: $10.1002 / a b .21601$

Published online XX Month Year in Wiley Online Library

(wileyonlinelibrary.com). 
1992), the NEO Personality Inventory-Revised (Costa \& McCrae, 1992), the Proactive Aggression dimension of the Reactive-Proactive Aggression Questionnaire (Raine et al., 2006), and a laboratory task (Bushman et al., 2009). However, the link between self-esteem and aggression is further complicated by the fact that high levels of selfesteem have been found to characterize both aggressive and non-aggressive individuals (Baumeister, Bushman, \& Campbell, 2000). These discrepancies may emerge for multiple reasons. First, methodological differences could partially explain these contradictory findings with regard to the type of sample being examined (e.g., clinical, offender, community), gender composition and age of participants (e.g., adolescents or adults), and assessment tools (e.g., questionnaires, interviews, official reports, laboratory tasks). Second, it has been proposed that contextual contingencies such as social inclusion/exclusion or mating competition may influence the connection between self-esteem and aggression (Kirkpatrick, Waugh, Valencia, \& Webster, 2002). Third, the conceptualization of both aggression (e.g., actual behavior or trait aggressiveness) and self-esteem varies across studies. For example, self-esteem instability has been found to be a unique predictor of aggression (Falkenbach, Howe, \& Falki, 2013) and to serve as a moderator of the relationship between self-esteem level and aggression (Webster, Kirkpatrick, Nezlek, Smith, \& Paddock, 2007; Zeigler-Hill, Enjaian, Holden, \& Southard, 2014), as well as between self-esteem level and both anger and hostility (Kernis, Grannemann, \& Barclay, 1989). Therefore, it may be that other variables may intervene in the relationship between trait self-esteem and aggression (e.g., Webster et al., 2007). Notably, a longitudinal study found that low self-esteem was no longer a significant predictor of subsequent violent offending after controlling for factors such as gender, ethnicity, maternal educational level, IQ, average family living standards during childhood, and problematic behavior at age 13 (e.g., attention problems, anxious withdrawn behavior, and conduct problems; Boden et al., 2007). Similarly, the longitudinal association between low self-esteem and hostility was reduced in magnitude after controlling for the same factors. Some authors have also argued that selfesteem may have an indirect association with aggression through other psychological mechanisms (Velotti, Elison, \& Garofalo, 2014). Indeed, intense negative emotional reactions involving unfavorable evaluations of the self may challenge the ability of individuals to regulate their emotions (Velotti et al., 2014). Empirical evidence has supported the link between self-esteem and deficits in emotion regulation (e.g., alexithymia; Sasai, Tanaka, \& Hishimoto, 2011; Yelsma, 1995). Nevertheless, research concerning the possibility that emotion dysregulation mediates the link between self-esteem and aggression is clearly lacking. Conceptually similar results have emerged which show that emotion dysregulation mediates the relationship between negative affect and physical aggression (Donahue, Goranson, McClure, \& Van Male, 2014), although this study only examined undergraduate students and did not consider other aspects of aggression.

Recent theories concerning Antisocial Personality Disorder (Bateman, Bolton, \& Fonagy, 2013; Gilligan, 2003) have argued that self-esteem threats are very upsetting to violent individuals and often provoke overwhelming feelings of shame. When emotion regulation strategies fail, these violent individuals may rely on aggressive acts as an attempt to restore their feelings of self-worth (Bateman et al., 2013; Gilligan, 2003; Velotti et al., 2014). We were interested in the possibility that pervasive difficulties with emotion regulation may provide at least a partial explanation for the connection between self-esteem and dispositional aggression (i.e., trait aggression).

The role of emotion dysregulation as an antecedent of aggression has been attracting considerable interest in clinical and forensic settings in recent years (McMurran \& Howard, 2009; Roberton, Daffern, \& Bucks, 2012; Scott, DiLillo, Maldonado, \& Watkins, 2015; Skripkauskaite et al., 2015). For instance, Scott et al. (2015) found that two maladaptive emotion regulation strategies (i.e., negative urgency and emotional suppression) predicted greater aggression among undergraduate students. Also with adolescents, emotion regulation difficulties were linked to proactive and reactive aggression and showed a longitudinal association with later proactive (but not reactive) aggression (Skripkauskaite et al., 2015). Furthermore, recent empirical evidence demonstrated that offenders with maladaptive emotion regulation reported a more extensive history of violent acts (Roberton, Daffern, \& Bucks, 2014) and that sadistic traits were related to several dimensions of emotion dysregulation (i.e., negative urgency, emotional nonacceptance, lack of emotional awareness, and poor distress tolerance; Velotti \& Garofalo, 2015). Another study demonstrated that aspects of emotion dysregulation-such as difficulties in refraining from impulsive behavior when emotionally upset-were related to chronic anger expression in offenders across different countries (i.e., Italy and Australia; Velotti et al., 2015b). Additionally, other aspects of emotion dysregulationsuch as difficulties engaging in goal-directed behavior when distressed and limited access to emotion regulation strategies-have been shown to have unique associations with aggression in young adults (Velotti, Casselman, Garofalo, \& McKenzie, 2015a). Finally, among offenders, difficulties in attending to upsetting emotions (i.e., lack of emotional awareness) were linked to a more severe history of aggressive behavior above and beyond 
the influence of trait anger and chronic anger expression (Roberton, Daffern, \& Bucks, 2015).

To the best of our knowledge, no studies have examined the mediating role of emotion dysregulation in the association between self-esteem level and aggression in offender populations. In an attempt to shed light on this topic, we sought to determine whether self-esteem had an indirect association with aggression that was mediated by emotion dysregulation in an offender population. It is proposed that low levels of self-esteem will weaken the capacity for emotion regulation by heightening the experience of negative affective states which, in turn, will trigger aggressive behavior. Thus, we expected to confirm the link between low self-esteem and aggression among offenders (e.g., Rosenthal et al., 2011; Walker \& Bright, 2009) but we expected this association to be mediated by difficulties in regulating emotions. Specifically, we refer to emotion dysregulation as a multidimensional construct involving the following: (i) a lack of awareness for and understanding of emotions; (ii) non-acceptance of emotional responses; (iii) failure to engage in goal-directed behavior and inability to abstain from impulsive behavior while experiencing emotional distress; and (iv) a limited access to effective emotion regulation strategies (Gratz \& Roemer, 2004). In order to strengthen the generalizability of our results, we explored the same model in a matched community sample. We expected that offenders would show lower levels of self-esteem, and higher levels of emotion dysregulation and aggressive tendencies, than community-dwelling individuals.

\section{METHOD}

\section{Participants}

Data were obtained from 153 male inmates (mean age $=41.78, S D=11.84$ ) across four prisons in Northern Italy and 197 male community participants (mean age $=38.88, S D=10.89$ ) who were recruited in two large Italian cities through self-referrals in response to advertisements asking potentially interested volunteers for psychological studies. Each prisoner was Caucasian and had been convicted of a violent crime (i.e., offenses involving physical violence toward others). Participants were asked to complete a series of measures designed to assess their self-esteem level, emotion regulation, and aggressive tendencies. For the offender sample, these measures were completed in individual or small group sessions that took place in a quiet room where inmates usually meet with prison educators. Similarly, community participants completed all questionnaires individually or in small groups. All participants provided their written informed consent after being introduced to the aim of the study and participated as volunteers (i.e., they were not compensated for their participation). Participants were assured that they could withdraw from the study at any time and have their responses removed from the database upon request. The Italian Ministry of Justice approved the procedure for the prisoners. Our procedures for the prisoners and the community members complied with the ethical guidelines of the Italian Association of Psychology.

\section{Measures}

Self-esteem level. An Italian translation of the Rosenberg Self-Esteem Scale (RSES; Rosenberg, 1965) was used to capture global self-esteem (e.g., "On the whole, I am satisfied with myself"). Participants completed this 10-item instrument according to how they generally felt about themselves. Responses were made on scales ranging from one (strongly disagree) to five (strongly agree). Previous research has found the RSES - and its Italian translation-to have adequate psychometric properties (Blascovich \& Tomaka, 1991; Prezza, Trombaccia, \& Armento, 1997). Reliability coefficients for the present study are detailed in Table I.

Emotion dysregulation. An Italian version of the Difficulties in Emotion Regulation Scale (DERS; Gratz \& Roemer, 2004) was used to capture troubles with emotion regulation. The DERS is a comprehensive measure of emotion dysregulation that consists of 36 items grouped in 6 dimensions: (i) nonacceptance of emotional responses (Nonacceptance; 6 items: "When I'm upset, I feel ashamed with myself for feeling that way"); (ii) difficulties engaging in goal-directed behavior when distressed, or poor distress tolerance (Goals; 5 items: "When I'm upset, I have difficulty getting work done"); (iii) impulse control difficulties under negative emotional arousal, or negative urgency (Impulse; 6 items: "When I'm upset, I become out of control"); (iv) lack of emotional awareness (Awareness; 6 items, all reverse-keyed: "When I'm upset, I acknowledge my emotions"); (v) limited access to effective emotion regulation strategies (Strategies; 8 items: "When I'm upset, I believe that there is nothing I can do to make myself feel better"); and (vi) lack of emotional clarity (Clarity; 5 items: "I am confused about how I feel"). Participants responded by indicating how often each statement applied to them using scales ranging from 1 (almost never) to 5 (almost always), with higher scores indicating greater emotion dysregulation. Previous research has found the DERS - and its Italian translation-to have adequate psychometric properties (Giromini, Velotti, de Campora, Bonalume, Zavattini, 2012; Gratz \& Roemer, 2004). As shown in previous studies (Garofalo \& Velotti, 2014; Soenke, Hahn, Tull, $\&$ Gratz, 2010), the DERS total score provides a reliable global index of overall emotion regulation difficulties 
TABLE I. Self-Esteem Level, Emotion Dysregulation, and Aggression: Reliability Coefficients, Descriptive Statistics, and Group Comparisons

\begin{tabular}{|c|c|c|c|c|c|c|c|c|c|}
\hline & \multicolumn{3}{|c|}{ Offender Sample $(N=153)$} & \multicolumn{3}{|c|}{ Community Sample $(N=197)$} & \multicolumn{3}{|c|}{ Whole Sample $(N=350)$} \\
\hline & $\alpha$ & $M$ & $S D$ & $\alpha$ & $M$ & $S D$ & $\alpha$ & $F$ & $\eta_{p}^{2}$ \\
\hline Self-esteem level & .80 & 30.65 & 6.09 & .87 & 32.61 & 5.06 & .84 & $12.82^{* * *}$ & .04 \\
\hline Emotion dysregulation (DERS total score) & .91 & 80.22 & 18.92 & .92 & 79.46 & 18.49 & .92 & 0.14 & .00 \\
\hline \multicolumn{10}{|l|}{ DERS dimensions $\mathrm{a}^{\mathrm{a}}$} \\
\hline Nonacceptance & .79 & 13.37 & 4.91 & .85 & 11.71 & 4.61 & .82 & $10.57^{* *}$ & .03 \\
\hline Goals & .69 & 11.82 & 4.09 & .86 & 12.67 & 4.52 & .79 & $3.26^{\dagger}$ & .01 \\
\hline Impulse & .82 & 10.99 & 4.37 & .84 & 11.15 & 4.39 & .83 & 0.11 & .00 \\
\hline Awareness & .62 & 14.51 & 4.09 & .67 & 14.87 & 4.05 & .65 & 0.66 & .00 \\
\hline Strategies & .84 & 14.92 & 5.77 & .88 & 14.90 & 5.82 & .86 & 0.00 & .00 \\
\hline Clarity & .73 & 9.33 & 3.62 & .78 & 9.32 & 3.37 & .76 & 0.00 & .00 \\
\hline \multicolumn{10}{|l|}{$A Q$ dimensions $^{\mathrm{b}}$} \\
\hline Physical aggression & .74 & 19.81 & 6.64 & .79 & 18.49 & 6.25 & .77 & $3.48^{\dagger}$ & .01 \\
\hline Verbal aggression & .55 & 14.19 & 3.71 & .62 & 14.93 & 3.46 & .58 & $3.89^{*}$ & .01 \\
\hline Anger & .52 & 15.78 & 4.73 & .76 & 16.10 & 5.16 & .67 & 0.45 & .00 \\
\hline Hostility & .66 & 19.64 & 5.82 & .79 & 18.00 & 5.92 & .74 & $5.76^{*}$ & .02 \\
\hline
\end{tabular}

DERS, difficulties in emotion regulation scale; AQ, aggression questionnaire; $F$, statistics based on one-way ANOVAs; $\eta_{p}^{2}$, Partial Eta squared, effect size measure $(.01=$ small effect; $.06=$ medium effect; $.13=$ large effect; Cohen, 1988).

${ }^{\dagger} P<.10 ;{ }^{*} P<.05 ;{ }^{* *} P<.01 ;{ }^{* * *} P<.001$.

aOne-way MANOVA Wilks' $\lambda=.93, P<.001, \eta_{p}^{2}=.07$.

'One-way MANOVA Wilks' $\lambda=.94, P<.001, \eta_{p}^{2}=.07$.

(Gratz \& Roemer, 2004). Reliability coefficients for the present study are shown in Table I.

Aggressive tendencies. An Italian version of the Aggression Questionnaire (AQ; Buss \& Perry, 1992) was used to capture the propensity toward aggression. The AQ captures aggressive tendencies across 29 items, and can be divided into the four unique subscales: physical aggression (9 items: "Once in a while I can't control the urge to strike another person."); verbal aggression (5 items: "I can't help getting into arguments when people disagree with me."); anger (7 items: "I sometimes feel like a powder keg ready to explode."); and hostility (8 items: "When people are especially nice, I wonder what they want."). Participants responded to each item by indicating how much each statement was characteristic of them using scales ranging from 1 (extremely uncharacteristic of me) to 5 (extremely characteristic of me). Previous research has found the $\mathrm{AQ}$ - and its Italian translation-to have adequate psychometric properties (Buss \& Perry, 1992; Fossati, Maffei, Acquarini, \& Di Ceglie, 2003). For this study, internal consistency coefficients are reported in Table I.

\section{RESULTS}

The means, standard deviations, and group differences (based on ANOVA/MANOVA designs) on all study variables are provided in Table I.

Consistent with our expectations, the offender sample reported lower levels of self-esteem than the community sample. As for emotion dysregulation and aggression, our hypotheses were only partially supported. The two groups did not differ in terms of overall emotion dysregulation, but offenders reported significantly higher scores on the Nonacceptance scale of the DERS. Conversely, a trend to a significant difference was found on the DERS Goals facet, with community participants reporting higher scores. Offenders reported significantly higher scores on Hostility and significantly lower scores on Verbal aggression than community participants. The difference in Physical aggression (with offenders scoring higher) only tended to significance. Intercorrelations among all measures are provided in Table II.

Inspection of the correlation matrix revealed that selfesteem level was negatively associated with emotion dysregulation, physical aggression, anger, and hostility in the offender sample. Emotion dysregulation was positively associated with physical aggression, anger, and hostility. It is important to note that neither selfesteem level nor emotion dysregulation was associated with verbal aggression in the offender sample, whereas an association between emotion dysregulation and verbal aggression occurred in the community sample. Besides this difference, the pattern of correlations was largely consistent across groups.

\section{Mediation Analyses}

We conducted a mediation analysis to test the proposed indirect effects model suggesting that the association 
between self-esteem level and aggressive tendencies may be due, at least in part, to emotion dysregulation. More specifically, we tested a mediation model with a single predictor, single mediator, and multiple outcomes (i.e., the four subscales of the AQ; see Fig. 1). To accomplish this, a bootstrapping approach was used (e.g., Hayes, 2009; MacKinnon, Lockwood, \& Williams, 2004; Preacher \& Hayes, 2004, 2008; Williams \& MacKinnon, 2008). Bootstrapping involves creating a repeated series of representations of the population by resampling from the current sample in an attempt to mimic the original sampling procedure. For the current study, we chose to set the number of bootstrapping samples to 5,000. In turn, these 5,000 bootstrapping samples were used to generate a $95 \%$ confidence interval for the indirect effect. The confidence interval generated from this process is considered to be statistically significant if it does not contain the value of zero. An SPSS Macro (i.e., PROCESS; Hayes, 2013) was used to conduct the mediation analysis.

Within the PROCESS macro, a seed command was implemented, which allowed us to test the model with multiple outcomes. This command is necessary, as the PROCESS macro is limited to testing models with a single predictor, a single mediator, and a single outcome. Therefore, we had to run a separate model for each of the four subscales of the AQ. However, the seed command links each of these four models by ensuring that each bootstrapping process begins with the same number which, in turn, causes the confidence intervals between the four models to adhere to the same bounds. Furthermore, because the same predictor and mediator variables were used across the models, the direct and indirect effects of the predictor on the four outcome variables will be the same if they are calculated separately or simultaneously (see Hayes, 2013, for an extended discussion). This approach was used in both the offender and the community sample.

Mediation analyses in the offender sample. The summary statistics for this model are presented in Table III, whereas a graphical depiction is provided in Figure 1.

The model explained a significant portion of the variance for each of the dependent variables, except verbal aggression. Specifically, the model explained: roughly $12 \%$ of the variance in physical aggression $\left(R^{2}=.11, \quad F[2,150]=9.63, P<.001\right) ; 24 \%$ of the variance in anger $\left(R^{2}=.24, \quad F[2,149]=23.15\right.$, $P<.001)$; and $11 \%$ of the variance in hostility $\left(R^{2}=.11, F[2,150]=9.40, P<.001\right)$. The model was not significant for verbal aggression $\left(R^{2}=.02, F\right.$ $[2,149]=1.37, P>.05)$. The total effects indicated that self-esteem level was negatively associated with physical aggression, anger, and hostility. However, no 

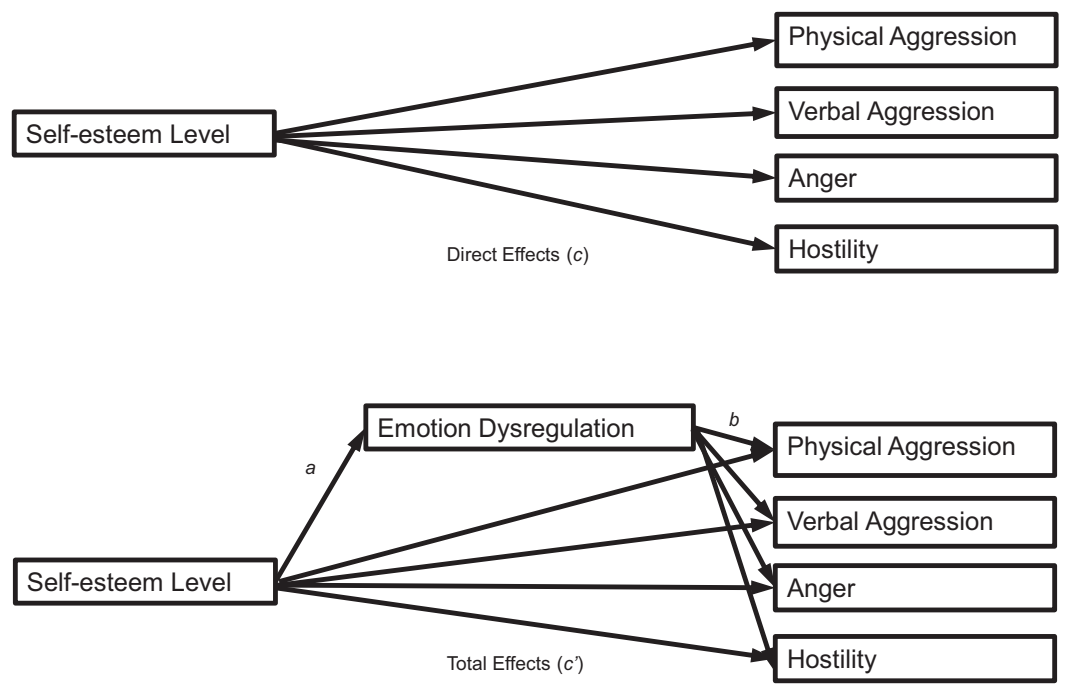

Fig. 1. An illustration of our mediation model including path labels. More specifically, we hypothesized that the relationship between self-esteem level and aggression would be mediated by emotion dysregulation. In the offender sample, significant results emerged for physical aggression, anger, and hostility, whereas all indirect effects were significant in the community sample (see Table III).

significant association occurred between self-esteem level and verbal aggression. Taken together, these results suggest that offenders with lower levels of selfesteem reported greater tendencies toward physical aggression, anger, and hostility. Furthermore, our mediation hypothesis was supported for all subscales except for verbal aggression. This suggests that there is an indirect effect of self-esteem level on certain aspects of aggression (i.e., physical aggression, anger, and hostility) through emotion dysregulation.

Follow-up analyses were conducted to examine the mediating role of specific dimensions of emotion dysregulation using the same procedures outlined above, and entering all DERS dimensions as simultaneous mediators in previously significant indirect effects. In the interest of parsimony, we only report those results that were statistically significant. Specifically, with selfesteem levels as the predictor variable, findings supported the unique mediating roles of Impulse (i.e., inability to control impulsive behavior when upset, or negative urgency) in physical aggression (point estimate $=-.20,95 \% \mathrm{CI}[-.39,-.07]$, overall $R^{2}=.21, F$ $[7,145]=5.57, P<.001)$ and anger (point estimate $=$ $-.11,95 \%$ CI $[-.21,-.03]$, overall $R^{2}=.37, F$ $[7,144]=11.83, P<.001)$. In contrast, none of the DERS scales uniquely accounted for the indirect effect of self-esteem on hostility, suggesting that this effect could be due to shared variance among DERS dimensions. Full mediation occurred in both cases (i.e., the direct effects were not significant, all $p \mathrm{~s}>.05$ ).

Mediation analyses in the community sample. The summary statistics for this model are presented in
Table III and a graphical depiction is provided in Figure 1. The model explained a portion of the variance for each of the dependent variables. In particular, the model explained: roughly $11 \%$ of the variance in physical aggression $\left(R^{2}=.11, \quad F[2,194]=11.55\right.$, $P<.001) ; 26 \%$ of the variance in anger $\left(R^{2}=.26, F\right.$ $[2,194]=34.81, P<.001) ; 38 \%$ of the variance in hostility $\left(R^{2}=.38, F[2,194]=58.30, P<.001\right)$; and $4 \%$ of the variance in verbal aggression $\left(R^{2}=.04, F\right.$ $[2,194]=4.52, P<.05)$. As was the case in the offender sample, the total effects indicated that self-esteem level was negatively associated with physical aggression, anger, and hostility, but not with verbal aggression. This suggests that community members with lower levels of self-esteem reported greater tendencies toward physical aggression, anger, and hostility. Similar mediation results emerged in the community sample. More specifically, full mediation was observed for physical aggression, verbal aggression, and anger. However, the effect of self-esteem level on hostility was only partially mediated by emotion regulation in the community sample. Furthermore, the emergence of full mediation for verbal aggression is unique to the community sample. This suggests that there are different indirect effects of self-esteem level on aggression in community members, and that there may be different processes at work in these individuals.

Further, as with the offender sample, we tested whether specific dimensions of emotion dysregulation uniquely accounted for the significant indirect effects of self-esteem on aggression dimensions. In the community sample, the results indicated the following mediations. 
Impulse (i.e., negative urgency) independently explained the associations that self-esteem level had with physical aggression (point estimate $=-.36,95 \%$ CI $[-.56,-.22]$, overall $R^{2}=.33, F[7,189]=13.04$, $P<.001$ ); verbal aggression (point estimate $=-.10$, $95 \%$ CI $[-.19,-.03]$, overall $R^{2}=.10, F[7,189]=3.01$, $P<.01$ ); and anger (point estimate $=-.31,95 \% \mathrm{CI}$ $[-.45,-.19]$, overall $R^{2}=.43, \quad F[7,189]=20.21$, $P<.001)$. Finally, both Impulse and Strategies (i.e., limited access to emotion regulation strategies) emerged as unique mediators in hostility, point estimate $=-.12$, $95 \% \mathrm{CI}[-.24,-.04]$, and point estimate $=-.16,95 \% \mathrm{CI}$ $[-.29,-.04]$, respectively, overall $R^{2}=.40, \quad F$ $(7,189)=17.60, P<.001$. Of note, the relative contribution of the DERS Impulse and Strategies scales did not differ significantly, $\Delta=.04,95 \%$ CI $[-.15, .20]$, indicating that the strength of the mediating effect through these dimensions was similar. Full mediation occurred in all cases (i.e., the direct effects were not significant, all $p \mathrm{~s}>.05)$.

\section{Alternative Mediation Model ${ }^{1}$}

In order to account for the possibility that some individuals may have higher levels of trait anger and trait hostility-and thus may be more inclined to physical aggression than others-additional models with multiple predictors and multiple mediators were assessed. That is, the affective (i.e., anger) and the cognitive (i.e., hostility) components of aggression may be conceptualized as precursors to physical aggression (i.e., the instrumental component). To examine this possibility, a model was constructed

\footnotetext{
${ }^{1}$ Two other alternative models were analyzed reversing the order of the variables from our original model (see Hayes, 2013, for an extended discussion of this process). More specifically, we first examined an alternative model where self-esteem level mediated the associations between emotion dysregulation and aggressive tendencies in order to compare the fit of these models with our proposed model. Our results suggested that this alternative model produced a fit similar to that of the model reported in the manuscript. However, the indirect effect of emotion dysregulation on aggression dimensions through self-esteem was only (marginally) significant as regards verbal aggression in the community sample. Then, we examined a second alternative model with aggression dimensions as simultaneous mediators in the association between selfesteem and emotion dysregulation. These models explained a greater portion of the variance in both the offender $\left(R^{2}=.39\right)$ and the community sample $\left(R^{2}=.49\right)$, as expected since it included more variables. However, mediation results revealed that, in the offender sample, only anger (and only partially) mediated the relation between self-esteem and emotion dysregulation. Similarly, in the community sample, only partial mediation occurred through anger and hostility. Although future research are encouraged to further address the viability of these alternative model, we decided to retain the original proposed model as the one which provided stronger results. Due to space limits, summary statistics for these alternative models are not reported in this paper, but are available upon request to the first author.
} 
wherein self-esteem level and emotion regulation (each controlling for the influence of the other) acted as predictors of physical aggression, while the components of anger and hostility acted as simultaneous mediators in both the offender and community samples. As was the case in the previous analyses, a seed command was employed in the PROCESS macro. However, in this analysis it was needed due to our use of multiple predictors.

Alternative model in the offender sample. The summary statistics for this model are presented in Table IV and a graphical depiction is provided in Figure 2. The entire model explained a substantial portion of the variance in physical aggression $\left(R^{2}=.49, \quad F[4,147]=34.94, \quad P<.001\right)$. The total effects in this model indicated that emotion dysregulation was positively associated with physical aggression when controlling for self-esteem levels, whereas a significant association did not occur for self-esteem level after controlling for the variance accounted for by emotion dysregulation. This suggests that offenders who have more difficulties in emotion regulation report greater tendencies to engage in physical aggression. Significant mediation results emerged in the offender sample. More specifically, both anger and hostility fully accounted for the association between emotion dysregulation and physical aggression. This suggests that trait anger and hostility may explain the association between emotion dysregulation and physical aggression. In particular, the difference between the two indirect effects was significant $(\Delta=.08,95 \%$ CI: .04-.13) which shows that the strength of the indirect effect through anger was larger than the one through hostility.

Alternative model in the community sample.

The summary statistics for this model are presented in Table IV and a graphical depiction is provided in Figure 2. The model explained a significant portion of the variance in physical aggression $\left(R^{2}=.46, F\right.$ $[4,192]=40.91, P<.001)$. As was the case in the offender sample, the total effects in this model indicated that emotion regulation was positively associated with physical aggression, but not with self-esteem level (when controlling for the variance shared between the two). This suggests that community members are similar to offenders in that those who have more difficulties in emotion regulation report greater tendencies toward physical aggression. Slightly different mediation effects emerged in the community sample. Indeed, only anger significantly mediated the effect of emotion regulation on physical aggression. Therefore, it is likely that there are different processes that lead offenders and community members to physical aggression. These findings also suggest that there may be complex interactions

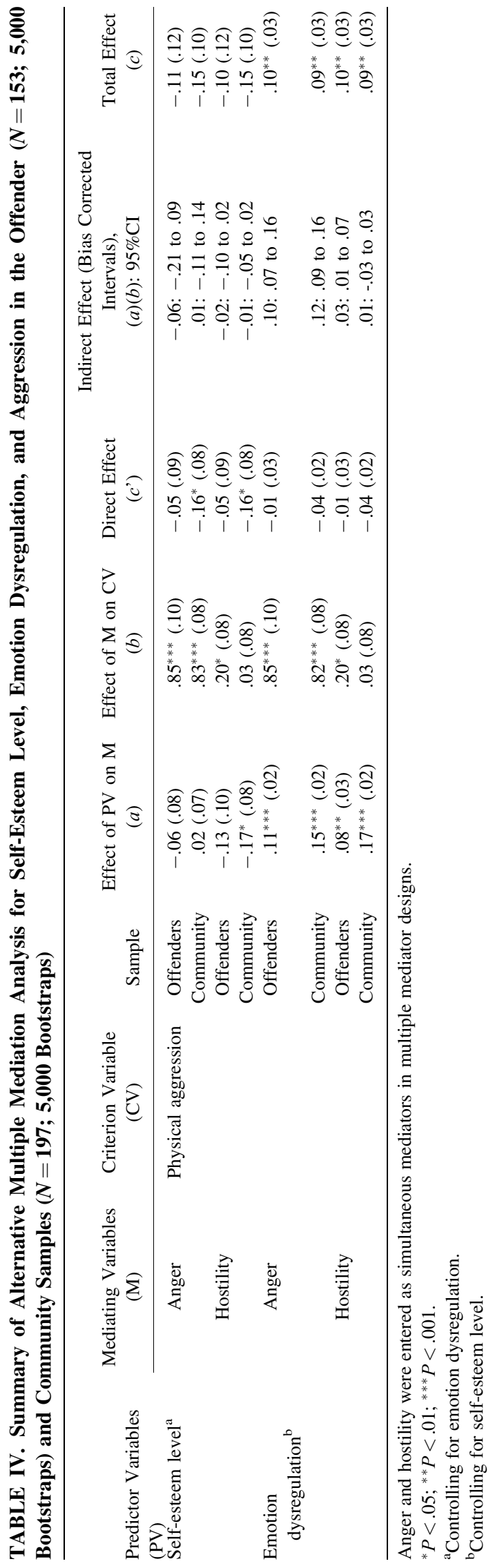



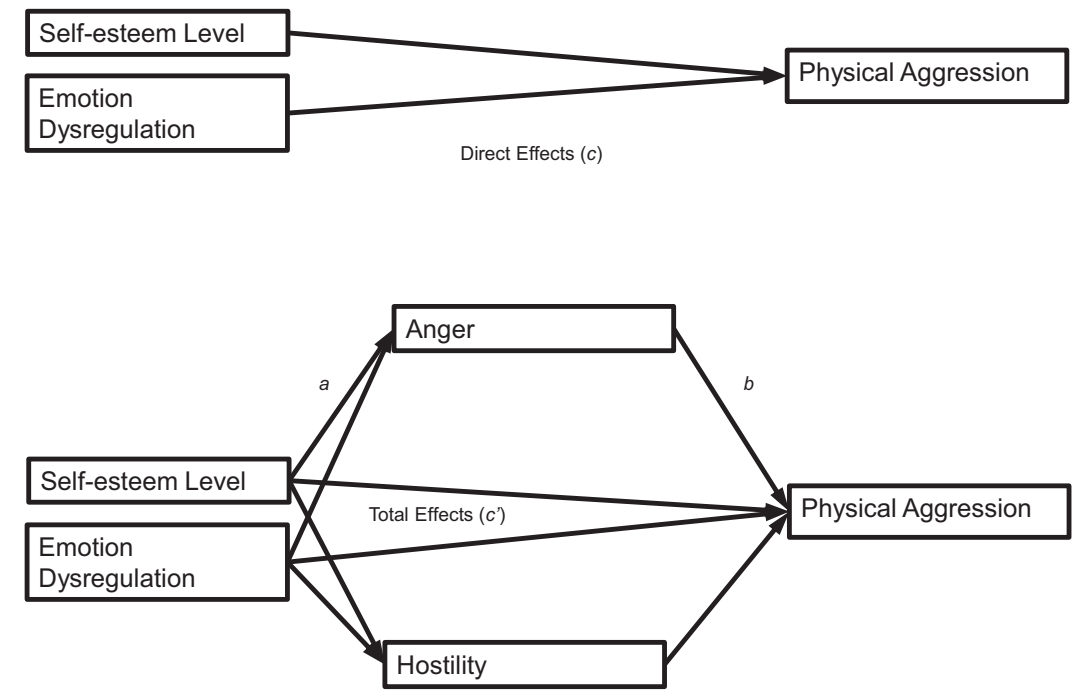

Fig. 2. An illustration of our alternative multiple mediation model including path labels. More specifically, we hypothesized that the relationship between self-esteem level, emotion dysregulation, and physical aggression would be mediated by anger and hostility. Significant results emerged for the indirect effect of emotion dysregulation (but not self-esteem) on physical aggression: through both anger and hostility among offenders, and through anger alone in the community sample (see Table IV).

between the traits that lead individuals to act in physically aggressive ways. ${ }^{2}$

\section{DISCUSSION}

Aggression researchers have often considered selfesteem as a possible trigger for violent and aggressive behavior. Additionally, interest has increased in the contribution of emotion dysregulation to aggression, yet research examining the joint role of self-esteem and emotion dysregulation is lacking. In the present study, we sought to confirm the proposed indirect effect of low self-esteem on aggression through the mediating role of emotion dysregulation in a sample of violent offenders and in a matched community sample.

Consistent with previous studies (e.g., Gilligan, 2003; Tangney, Stuewig, \& Hafez, 2011; Velotti et al., 2015b), we found that offenders showed significantly lower levels of self-esteem than community participants. Regarding emotion dysregulation and aggression, our results support the importance of considering these aspects at a facet level. Indeed, offenders featured selective difficulties in emotional nonacceptance (as indicated by a significantly higher score on this dimension than the community sample), whereas no differences occurred when examining the overall level

\footnotetext{
${ }^{2}$ As offenders were significantly older than community participants, $t$ $(341)=2.36, P<.05,95 \%$ CI $[.48,5.32]$, all analyses reported in the Results section were repeated controlling for participants' age, and all findings remained unchanged.
}

of emotion dysregulation. This finding seems consistent with extant theories (e.g., Bateman et al., 2013) attesting to the role of mentalization deficits as a likely trigger of violent behavior. Accordingly, difficulties in being able to think of and reflect on inner experiences and feelings assuming a nonjudgmental stance, could undermine offenders' ability to regulate emotions before they become overwhelming which, in turn, may lead to violent behavior (Velotti \& Garofalo, 2015). Our inmate sample did not report elevated levels for all of the aggression dimensions. Rather, offenders only reported relatively high levels of hostility which suggests that hostility may be characteristic of their psychological functioning. Consistent with previous seminal works (e.g., Nestor, 2002), this finding seems to suggest that a tendency to perceive the outside world as threatening and menacing (and respond accordingly with hostility) could characterize the mental functioning of offenders, representing a strong predictor of violent behavior. This was also corroborated by the alternative mediation model we tested in which-among offenders-hostility accounted for the association between emotion dysregulation and physical aggression. Finally, we were surprised to find only marginally higher levels of physical aggression for the offenders than the community sample. This may either depend on the offenders' tendency to present themselves in a socially desirable fashion, or may suggest that other components of aggression (e.g., a hostile cognitive style) characterize offenders more than the behavioral components. Also, it could suggest that rather than being a matter of 
frequency, the difference between physical aggressive acts committed by violent offenders and communitydwelling individuals could be more tightly linked to issues of severity.

Our results concerning group comparisons seem to suggest that investigating global indices could be misleading. For example, community participants reported greater levels of verbal aggression than the offender group (which could be considered as a more adaptive way to express aggressive attitudes; Buss \& Perry, 1992), and more difficulties in persisting in goaldirected behavior when experiencing negative emotions. This latter finding could be interpreted in light of the fact that violent offenders are likely to experience negative emotions more often (Garofalo, 2015; Velotti et al., $2015 b$ ). As a result, they may develop a stronger ability to experience emotional distress and persist in the pursuit of their own goals (including those that are motivated by antisocial tendencies). Overall, our results corroborated the proposed model, expanding extant evidence on the central role of emotion dysregulation in the study of aggression. We first confirmed the association between low self-esteem and aggression as reported in previous studies with offenders (e.g., Walker \& Bright, 2009) which is consistent with the results of a recent meta-analysis (Rosenthal et al., 2011). Furthermore, we found support for the role of emotion dysregulation in mediating the association between low self-esteem and aggression. Interestingly, three out of four mediational models were significant in the offender sample. More specifically, low self-esteem had indirect associations with higher levels of physical aggression, anger, and hostility via emotion dysregulation. Thus, we confirmed previous evidence of the link between emotion dysregulation and the affective component of aggression (i.e., anger; Velotti et al., 2015b). Furthermore, the current study integrates this finding with the association between difficulties in emotion regulation and physical aggression (as reported in student samples; e.g., Donahue et al., 2014). Moreover, emotion dysregulation played a role in explaining the relationship between low self-esteem and the cognitive component of aggression, namely hostility. In other words, offenders with lower levels of selfesteem seemed to be more prone to hostile thoughts and reported perceiving the outside world as malevolent and threatening. This relationship was explained by emotion dysregulation, suggesting that low self-esteem alone cannot explain hostile thoughts, and that an inability to deal with negative affect may be important. Therefore, individuals who have difficulties in regulating negative emotions may attribute their suffering to external situations or events, or even to a specific person in their environment. Considering other people as menacing and untrustworthy could ultimately make aggressive and violent behavior easier (Nestor, 2002). When examining the role of specific emotion dysregulation dimensions, we found that negative urgency (i.e., a difficulty refraining from impulsive behavior when under negative emotional arousal) uniquely accounted for the indirect effect of self-esteem on physical aggression and anger. This is in line with the acknowledged role of impulsivity as a contributing factor to violent behavior (Nestor, 2002). Also, it is consistent with a mentalizing framework (Bateman et al., 2013) emphasizing that an underlying difficulty in thinking about and reflecting upon negative feelings may make these individuals feel compelled to act out their impulse toward others.

The fact that self-esteem and emotion dysregulation were not associated with verbal aggression for the offender sample is somewhat surprising. However, it is important to note that previous research (e.g., Buss \& Perry, 1992; Fossati et al., 2003) reported weak correlations between verbal aggression and other dimensions of the aggression domain, and no associations between specific facets of emotion dysregulation and verbal aggression in community-dwelling young adults (e.g., Velotti et al., 2015a). Further, verbal aggression has been found to be associated with healthy aspects of psychological functioning (e.g., assertiveness; Buss \& Perry, 1992). Another possible explanation for the lack of associations that verbal aggression had with self-esteem and emotion dysregulation is that the verbal aggression scale of the AQ often yields weak internal consistency coefficients (e.g., Fossati et al., 2003). When developing their measure, Buss and Perry (1992) also stated that verbal aggression was difficult to categorize because of its ambiguity. For example, irony or sarcasm could sometimes be considered as verbally aggressive even though they do not inherently imply an aversive intent as aggression does. Also, the AQ items aimed at capturing verbal aggression measure the tendency to be an argumentative person, rather than assessing different forms of verbal aggression such as abusive and threatening behavior (e.g., shouting and swearing at someone). Related to these concerns, other authors have proposed that verbal aggression may be less related to difficulties in emotion regulation and more related to other factors (e.g., insecure adult attachment; Gormely \& Lopez, 2010). All these considerations received further, albeit indirect, support by the different pattern which characterized the community sample of the present study, as well as by the significantly higher score on verbal aggression reported by the community sample.

Indeed, among community participants, the indirect effect of low self-esteem through emotion dysregulation was significant for all aggression dimensions, including 
verbal aggression. Notably, the variance explained in hostility was higher in the community sample (when compared to the offender sample), suggesting that the relevance of self-esteem level and emotion dysregulation for this dimension of aggression may be relatively more important at sub-clinical manifestations of aggressive tendencies. In other terms, other factors may contribute more decisively in explaining the hostile attitudes among offenders (e.g., environmental influences).

When exploring the role of selected aspects of emotion dysregulation in explaining the association between low self-esteem and aggression among community members, negative urgency emerged as a unique and independent mediator. This seems to support the idea that prevention and treatment modules should target this aspect of emotion dysregulation as a means of reducing aggressive tendencies. In the community sample, an inability to rely on effective emotion regulation strategies also mediated the link between low selfesteem and trait hostility. This interesting finding seems to suggest the following scenario. Among community participants (i.e., at sub-clinical levels of violent behavior), low self-esteem may contribute to a belief that nothing can be done to feel better when experiencing negative feelings. In turn, this lack of confidence in the ability to regulate emotions (what is actually captured by the DERS Strategies scale) may lead to the development of a hostile-oriented cognitive style, in which others are considered as untrustworthy or even threatening. The only way that these individuals may feel capable of dealing with these perceived threats is to respond with hostility and aggressive behavior.

The alternative models that we tested support the importance of hostility in understanding self-esteem and aggressive behavior. Specifically, when considering anger and hostility as potential psychological bridges that link self-esteem and emotion dysregulation with physical aggression (i.e., the instrumental component of aggression), different patterns emerged in the two samples. Among offenders, both anger and hostility mediated the association between emotion dysregulation and physical aggression, whereas in the community sample, only anger accounted for this relation. This seems particularly important as offenders showed significantly higher levels of hostility than the comparison sample, suggesting that hostility may represent a crucial component for both understanding their violent behavior and for tailoring treatment programs.

\section{Limitations}

Despite their relevance, the present findings should be considered in light of the limitations of this study. For example, all variables were assessed through self-report, which can result in inflated correlations due to shared method variance, and are prone to influences from social desirability, poor insight, or deception. Also, the internal consistencies of some of the subscales were quite low. This was not unexpected, given the limited length of each scale (Cronbach's $\alpha$ depends on both average interitem correlation and scale length; Nunnally \& Bernstein, 1994) although the internal consistencies are consistent with those reported for previous studies that have used the Italian versions of the AQ (e.g., Fossati et al., 2003) and the DERS (e.g., Velotti \& Garofalo, 2015). It should also be acknowledged that low reliability attenuates correlations; hence, the relatively low reliability coefficients in the present study places our findings on the conservative side, rather than producing overestimation (i.e., false positive results). Another potential limitation of our study was that the cross-sectional design does not allow assessment of causality. As a result, it could be argued that other theoretical approaches could lead to a different ordering of the variables in our mediation analyses. More specifically, sociometer theory (e.g., Leary \& Baumeister, 2000; Leary, Terdal, Tambor, \& Downs, 1995) suggests that self-esteem functions as a monitor of how an individual is doing interpersonally. That is, if an individual is highly regarded and valued by others in their social environment, then he or she should experience relatively high levels of self-esteem (Leary \& Baumeister, 2000; Leary et al., 1995). Therefore, if an individual does something that could cause them to lose favor with their friends and relatives (e.g., act aggressively), then it may lead to the individual experiencing a drop in feelings of self-worth when receiving feedback regarding his or her actions. Taking this into consideration, it could be argued that it may be best to treat self-esteem as a criterion variable. To explore this possibility, the corresponding models were assessed. However, for the sake of parsimony, and due to their weaker findings, these models were not included.

Another limitation is that the extent to which we can specify the directionality of effects is limited by the crosssectional nature of the study (Bullock, Green, \& Ha, 2010; MacKinnon, Krull, \& Lockwood, 2000). We attempted to limit the risk of misinterpretation by testing alternative models but this approach does not compensate for our reliance on cross-sectional data. Moreover, we only focused on self-esteem level in the present study. As a result, we suggest that future studies include measures such as self-esteem instability, which are likely to moderate the effects of self-esteem level (Kernis et al., 1989; Webster et al., 2007; Zeigler-Hill et al., 2014) and to differentially predict aggression dimensions (Lee, 2014). Additionally, an interesting extension of this work could be to explore whether emotion dysregulation also mediates the relationship between fragile self-esteem and 
aggression. Indeed, when they experience threats to their reputation, individuals with fragile self-esteem may experience intense shame which may be similar to the experiences of people with low self-esteem (Elison, Garofalo, \& Velotti, 2014) which may motivate them to act in more aggressive ways (Webster et al., 2007; Zeigler-Hill et al., 2014) or become increasingly hostile (Kernis et al., 1989). Finally, it should be noted that although we have tested various alternative models, the possibility of suppressor or confounding effects cannot be entirely ruled out.

\section{CONCLUSIONS}

The present study is the first to examine the mediating role of emotion dysregulation in explaining the association between self-esteem level and aggression. We found empirical support for the indirect effect (as opposed to the proposed direct effect) of self-esteem level on aggression, helping to elucidate the mixed findings concerning this relationship in previous studies. Then, we confirmed the relevance of emotion dysregulation as a risk factor for aggression, in line with previous studies (e.g., Gilligan, 2003; Nestor, 2002; Roberton et al., 2012, 2014, 2015; Scott et al., 2015; Skripkauskaite et al., 2015). Furthermore, we extended existing evidence with community samples (e.g., Donahue et al., 2014) to violent offenders, showing that here too emotion dysregulation mediates the association between self-esteem and aggression.

\section{ACKNOWLEDGMENTS}

All authors certify that there is no conflict of interest with any financial organization regarding the material discussed in the manuscript. The authors wish to thank the Department of Corrections for the Region of Liguria and the prison staff for their active cooperation.

\section{REFERENCES}

Bateman, A., Bolton, R., \& Fonagy, P. (2013). Antisocial personality disorder: A mentalizing framework. FOCUS: The Journal of Lifelong Learning in Psychiatry, 11, 178-186. doi: 10.1176/appi. focus.11.2.178

Baumeister, R. F., Bushman, B. J., \& Campbell, W. K. (2000). Selfesteem, narcissism, and aggression: Does violence result from low self-esteem or from threatened egotism? Current Directions in Psychological Science, 9(1), 26-29. doi: 10.1111/1467-8721.00053

Boden, J. M., Fergusson, D. M., \& Horwood, L. J. (2007). Self-esteem and violence: Testing links between adolescent self-esteem and later hostility and violent behavior. Social Psychiatry and Psychiatric Epidemiology, 42, 881-891. doi: 10.1007/s00127-007-0251-7

Bullock, J. G., Green, D. P., \& Ha, S. E. (2010). Yes, but what's the mechanism? (Don't expect an easy answer). Journal of Personality and Social Psychology, 98, 550-558. doi: 10.1037/a0018933
Bushman, B. J., \& Anderson, C. A. (2001). Is it time to pull the plug on the hostile versus instrumental aggression dichotomy? Psychological Review, 108, 273-279. doi: 10.1037/0033-295X.108.1.273

Bushman, B. J., Baumeister, R. F., Thomaes, S., Ryu, E., Begeer, S., \& West, S. G. (2009). Looking again, and harder, for a link between low self-esteem and aggression. Journal of Personality, 77, 427-446. doi: 10.1111/j.1467-6494.2008.00553.x

Buss, A. H., \& Perry, M. (1992). The aggression questionnaire. Journal of Personality and Social Psychology, 63, 452-459. doi: 10.1037/00223514.63.3.452

Cohen, J. (1988). Statistical power analysis for the behavioural sciences, 2nd ed.). New York: Academic Press.

Costa, P. T., Jr., \& McCrae, R. R. (1992). Revised NEO personality iventory (NEO-PI-R) and NEO five-factor inventory (NEO-FFI) professional manual. Odessa: Psychological Assessment Resources.

Donahue, J. J., Goranson, A. C., McClure, K. S., \& Van Male, L. M. (2014). Emotion dysregulation, negative affect, and aggression: A moderated, multiple mediator analysis. Personality and Individual Differences, 70, 23-28. doi: 10.1016/j.paid.2014.06.009

Donnellan, M. B., Trzesniewski, K. H., Robins, R. W., Moffitt, T. E., \& Caspi, A. (2005). Low self-esteem is related to aggression, antisocial behavior, and delinquency. Psychological Science, 16, 328-335. doi: 10.1111/j.0956-7976.2005.01535.x

Elison, J., Garofalo, C., \& Velotti, P. (2014). Shame and aggression: Theoretical considerations. Aggression and Violent Behavior, 19, 447 453. doi: 10.1016/j.avb.2014.05.002

Falkenbach, D. M., Howe, J. R., \& Falki, M. (2013). Using self-esteem to disaggregate psychopathy, narcissism, and aggression. Personality and Individual Differences, 54(7), 815-820. doi: 10.1016/j. paid.2012.12.017

Fossati, A., Maffei, C., Acquarini, E., \& Di Ceglie, A. (2003). Multigroup confirmatory component and factor analyses of the Italian version of the aggression questionnaire. European Journal of Psychological Assessment, 19, 54-65. doi: 10.1027//1015-5759.19.1.54

Garofalo, C. (2015). Emozionalità negativa ed autostima in un campione di offender detenuti: Uno studio preliminare [Negative emotionality and self-esteem in an incarcerated offender sample: Preliminary investigation]. Giornale Italiano di Psicologia [Italian Journal of Psychology], 42, 385-393. ISSN: 0390-5349.

Garofalo, C., \& Velotti, P. (2014). Alcohol misuse in psychiatric patients and nonclinical individuals: The role of emotion dysregulation and impulsivity. Addiction Research and Theory, 23, 294-300. doi: 10.3109/16066359.2014.987758

Gilligan, J. (2003). Shame, guilt, and violence. Social Research, 70, 1149-1166.

Giromini, L., Velotti, P., de Campora, G., Bonalume, L., \& Zavattini, G. C. (2012). Cultural adaptation of the difficulties in emotion regulation scale: Reliability and validity of an Italian version. Journal of Clinical Psychology, 68, 989-1007. doi: 10.1002/jclp.21876

Gormely, B., \& Lopez, F. G. (2010). Psychological abuse perpetration in college dating relationships: Contributions of gender, stress and adult attachment orientations. Journal of Interpersonal Violence, 25, 204 218. doi: $10.1177 / 0886260509334404$

Gratz, K. L., \& Roemer, L. (2004). Multidimensional assessment of emotion regulation and dysregulation: Development, factor structure and initial validation of the difficulties in emotion regulation scale. Journal of Psychopathology and Behavioral Assessment, 26, 41-54. doi: 10.1023/B:JOBA0000007455.08539.94

Hayes, A. F. (2009). Beyond Baron and Kenny: Statistical mediation analysis in the new millennium. Communication Monographs, 76 , 408-420. doi: 10.1080/03637750903310360

Hayes, A. F. (2013). Introduction to mediation, moderation, and conditional process analysis: A regression-based approach. New York: Guilford Press. 
Hoeve, M., Colins, O. F., Mulder, E. A., Loeber, R., Stams, G. J. J. M., \& Vermeiren, R. R. J. M. (2015). The association between childhood maltreatment, mental health problems, and aggression in justiceinvolved boys. Aggressive Behavior, Advance online publication doi: 10.1002/ab.21586

Leary, M. R., Tambor, E. S., Terdal, S. K., \& Downs, D. L. (1995). Selfesteem as an interpersonal monitor: The sociometer hypothesis. Journal of Personality and Social Psychology, 68, 518-530. doi: 10.1037/0022-3514.68.3.518

Leary, M. R., \& Baumeister, R. F. (2000). The nature and function of selfesteem: Sociometer theory. Advances in Experimental Social Psychology, 32, 1-62. doi: 10.1016/S0065-2601(00)80003-9

Lee, E. J. (2014). The relationship between unstable self-esteem and aggression differences in reactive and proactive aggression. Journal of Early Adolescence, 34, 1075-1093. doi: 10.1177/0272431613518973

Kernis, M. H., Grannemann, B. D., \& Barclay, L. C. (1989). Stability and level of self-esteem as predictors of anger arousal and hostility. Journal of Personality and Social Psychology, 56 (6), 1013-1022. doi: 10.1037/0022-3514.56.6.1013

Kirkpatrick, L. A., Waugh, C. E., Valencia, A., \& Webster, G. D. (2002). The functional domain specificity of self-esteem and the differential prediction of aggression. Journal of Personality and Social Psychology, 82, 756-767. doi: 10.1037/0022-3514.82.5.756

MacKinnon, D. P., Krull, J. L., \& Lockwood, C. M. (2000). Equivalence of the mediation, confounding and suppression effect. Prevention Science, 1, 173-181. doi: 10.1023/A:1026595011371

MacKinnon, D. P., Lockwood, C. M., \& Williams, J. (2004). Confidence limits for the indirect effect: Distribution of the product and resampling methods. Multivariate Behavioral Research, 39, 99128. doi: 10.1207/s15327906mbr3901_4

McMurran, M., \& Howard, R. C. (2009). Personality, personality disorder, and violence. New York: Wiley.

Muller, D., Bushman, B. J., Subra, B., \& Ceaux, E. (2012). Are people more aggressive when they are worse off or better off than others? Social Psychological and Personality Science, 3, 754-759. doi: 10.1177/1948550612436984

Nestor, P. G. (2002). Mental disorder and violence: personality dimensions and clinical features. American Journal of Psychiatry, 159, 1973-1978. doi: 10.1176/appi.ajp.159.12.1973

Nunnally, J. C., \& Bernstein, I. H. (1994). Psychometric theory (3rd ed). New York: McGraw Hill.

Preacher, K., \& Hayes, A. (2008). Asymptotic and resampling strategies for assessing and comparing indirect effects in multiple mediator models. Behavior Research Methods, 40, 879-891. doi: 10.3758/ BRM.40.3.879

Prezza, M., Trombaccia, F. R., \& Armento, L. (1997). La scala dell'autostima di Rosenberg: Traduzione e validazione Italiana. [The Rosenberg self-esteem Scale: Italian translation and validation.]. Bollettino di Psicologia Applicata [Applied Psychology Bulletin], 223, 35-44.

Raine, A., Dodge, K., Loeber, R., Gatzke-Kopp, L., Lynam, D., Reynolds, C., ... Liu, J. (2006). The reactive-proactive aggression questionnaire: Differential correlates of reactive and proactive aggression in adolescent boys. Aggressive Behavior, 32 (2), 159-171. doi: 10.1002/ab.20115

Roberton, T., Daffern, M., \& Bucks, R. S. (2012). Emotion regulation and aggression. Aggression and Violent Behavior, 17, 72-82. doi: 10.1016/ j.avb.2011.09.006

Roberton, T., Daffern, M., \& Bucks, R. S. (2014). Maladaptive emotion regulation and aggression in adult offenders. Psychology, Crime \& Law, 20, 933-954. doi: 10.1080/1068316X2014.893333

Roberton, T., Daffern, M., \& Bucks, R. S. (2015). Beyond anger control: Difficulty attending to emotions also predicts aggression in offenders. Psychology of Violence, 5, 74-83. doi: 10.1037/a0037214
Rosenberg, M. (1965). Society and the adolescent self-image. Princeton, NJ: Princeton University Press.

Rosenthal, S. A., Montoya, R. M., Ridings, L. E., Rieck, S. M., \& Hooley, J. M. (2011). Further evidence of the Narcissistic personality inventory's validity problems: A meta-analytic investigationResponse to Miller, Maples, and Campbell (this issue). Journal of Research in Personality, 45, 408-416. doi: 10.1016/j.jrp.2011.06.004

Sasai, K., Tanaka, K., \& Hishimoto, A. (2011). Alexithymia and its relationships with eating behavior, self esteem, and body esteem in college women. The Kobe Journal of Medical Sciences, 56, E231E238. doi: 10.1016/j.eatbeh.2006.11.005

Scott, J. P., DiLillo, D., Maldonado, R. C., \& Watkins, L. E. (2015). Negative urgency and emotion regulation strategy use: Associations with displaced aggression. Aggressive Behavior, Advance online publication doi: 10.1002/ab.21588

Skripkauskaite, S., Hawk, S. T., Branje, S. J. T., Koot, H. M., van Lier, P. A. C., \& Meeus, W. (2015). Reactive and proactive aggression: Differential links with emotion regulation difficulties, maternal criticism in adolescence. Aggressive Behavior, 41(3), 214-226. doi: 10.1002/ab.21583

Soenke, M., Hahn, K. S., Tull, M. T., \& Gratz, K. L. (2010). Exploring the relationship between childhood abuse and analogue generalized anxiety disorder: The mediating role of emotion dysregulation. Cognitive Therapy and Research, 34, 401-412. doi: 10.1007/s10608009-9264-8

Stanford, M. S., Houston, R. J., Mathias, C. W., Villemarette-Pittman, N. R., Helfritz, L. E., \& Conklin, S. M. (2003). Characterizing aggressive behavior. Assessment, 10, 183-190. doi: 10.1177/ 1073191103010002009

Tangney, J. P., Stuewig, J., \& Hafez, L. (2011). Shame, guilt and remorse: Implications for offender populations. Journal of Forensic Psychiatry and Psychology, 22, 706-723. doi: 10.1080/14789949.2011.617541

Velotti, P., Elison, J., \& Garofalo, C. (2014). Shame and aggression: Different trajectories and implications. Aggression and Violent Behavior, 19, 454-461. doi: 10.1016/j.avb.2014.04.011

Velotti, P., Casselman, R. B., Garofalo, C., \& McKenzie, M. D. (2015a). Emotion dysregulation and aggression among young adults in the U.S. A. and Italy: A cross-cultural comparison. In press.

Velotti, P., Garofalo, C., Callea, A., Bucks, R. S., Roberton, T., \& Daffern, M. (2015b). Exploring anger among offenders: Cross-cultural comparison of the role of emotion dysregulation and alexithymia. In press.

Velotti, P., \& Garofalo, C. (2015). Personality styles in a non-clinical sample: The role of emotion dysregulation and impulsivity. Personality and Individual Differences, 79, 44-49. doi: 10.1016/j. paid.2015.01.046

Walker, J. S., \& Bright, J. A. (2009). False inflated self-esteem and violence: a systematic review and cognitive model. The Journal of Forensic Psychiatry \& Psychology, 20, 1-32. doi: 10.1080/ 14789940701656808

Webster, G. D., Kirkpatrick, L. A., Nezlek, J. B., Smith, C. V., \& Paddock, E. L. (2007). Different slopes for different folks: Self-esteem instability and gender as moderators of the relationship between self-esteem and attitudinal aggression. Self and Identity, 6 (1), 74-94. doi: $10.1080 / 15298860600920488$

(2008). Resampling and distribution of the product methods for testing indirect effects in complex models. Structural Equation Modeling, 15, 23-51. doi: 10.1080/10705510701758166

Yelsma, P. (1995). Self-esteem and alexithymia. Psychological Reports, 77, 735-738. doi: 10.2466/pr0.1995.77.3.735

Zeigler-Hill, V., Enjaian, B., Holden, C. J., \& Southard, A. C. (2014). Using self-esteem instability to disentangle the connection between self-esteem level and perceived aggression. Journal of Research in Personality, 49, 47-51. 10.1016/j.jrp.2014.01.003 